\title{
Entropy Maximization, Cutoff Distribution, and Finite Stellar Masses
}

\author{
Ritesh Kumar Dubey, V. J. Menon, M. K. Pandey, and D. N. Tripathi \\ Department of Physics, Banaras Hindu University, Varanasi 221 005, India \\ Correspondence should be addressed to Ritesh Kumar Dubey, dubeyrk@rediffmail.com
}

Received 18 April 2008; Revised 16 July 2008; Accepted 26 August 2008

Recommended by Giovanni Carraro

\begin{abstract}
Conventional equilibrium statistical mechanics of open gravitational systems is known to be problematical. We first recall that spherical stars/galaxies acquire unbounded radii, become infinitely massive, and evaporate away continuously if one uses the standard Maxwellian distribution $f^{B}$ (which maximizes the usual Boltzmann-Shannon entropy and hence has a tail extending to infinity). Next, we show that these troubles disappear automatically if we employ the exact most probable distribution $f$ (which maximizes the combinatorial entropy and hence possesses a sharp cutoff tail). Finally, if astronomical observation is carried out on a large galaxy, then the Poisson equation together with thermal de Broglie wavelength provides useful information about the cutoff radius $r_{K}$, cutoff energy $\varepsilon_{K}$, and the huge quantum number $K$ up to which the cluster exists. Thereby, a refinement over the empirical lowered isothermal King models, is achieved. Numerically, we find that the most probable distribution (MPD) prediction fits well the number density profile near the outer edge of globular clusters.
\end{abstract}

Copyright (C) 2008 Ritesh Kumar Dubey et al. This is an open access article distributed under the Creative Commons Attribution License, which permits unrestricted use, distribution, and reproduction in any medium, provided the original work is properly cited.

\section{Introduction}

It is well known that standard methods $[1,2]$ of equilibrium statistical mechanics run into conceptual difficulties when applied to gravitational bound systems [3-11]. Basically, these troubles arise due to the peculiar behaviour of the gravitational interaction (either the pair potential or the mean field) at short or long distances. The aim of the present paper is to focus attention on the triple problems, namely, unbounded radius, infinite mass, and continuous evaporation of every stellar/galactic system described by the conventional Maxwell-Boltzmann (hereinafter referred to as the $\mathrm{MB})$ distribution.

Section 2 below points out that since the MB function $f^{B}$ maximizes only the simple-minded BoltzmannShannon entropy, its tail becomes illogical in the energy cells of small occupancy. The ensuing problems of the Maxwellian distribution cannot be really overcome by using ad hoc prescriptions such as enclosing the system in a hypothetical box [7] or modifying the Maxwellian form empirically by invoking gravitational tidal cutoff $[4,8]$. Next, Section 3 presents a detailed derivation of our most probable distribution (MPD) $f$ by taking hints from a preliminary investigation by Menon and Agrawal [12] in the molecular context and by Menon et al. [13] in the cosmological context. Such $f$ maximizes rigorously the more sophisticated combinatorial entropy and the corresponding variational conditions dictate that $f$ must possess a sharply truncated tail. Next, Section 4 demonstrates how our MPD idea applied to cosmology resolves the aforesaid troubles of the MB formalism, and how the Poisson equation brings additional features into our theory. We feel that the MPD philosophy may have bright applicational prospects in fitting cosmological data such as the classic study of stellar number densities in globular clusters done by King [14, Figure 2] and the important measurements performed by van Loon et al. [15, Figure 6] showing velocity distribution on the postmail-sequence stars in $\omega$ Centauri. Finally, the paper ends by presenting several concluding remarks in Section 5 where some other approaches to the subject (namely, self-consistent Hartree calculations, incomplete relaxation in low-density tail, canonical ensemble treatment of virialization, occurrence of a stellar mass spectrum in real gravitating systems, etc.) are also mentioned. 
Some related aspects of algebraic interest are reported in two useful appendices. Careful study of Sections 2 and 3 will reveal that quantum mechanical discretization of the single-particle levels is very convenient for setting up the combinatorial entropy and in finding the cutoff number; hence for the sake of ready reference we collect in Appendix A several known formulae concerning semiclassical one-body spectrum as well as the energy cell occupation number $v$. Also, a detailed treatment of our variational conditions in Section 4 requires that $\nu$ ! be replaced by $\Gamma(\nu+1)$ everywhere (even for $v \ll 1$ ); hence Appendix B tells why derivatives of factorials or gamma functions can be readily taken even in the cells of small occupation numbers.

\section{Difficulties with the MB Distribution}

\subsection{Preliminaries}

This section begins by quickly recalling a standard derivation of the famous Maxwell-Boltzmann distribution $f^{B}$ in equilibrium statistical mechanics. Particles are assumed to be moving in $D$ spatial dimensions at temperature $T$ under the influence of a mean field potential energy $W(r)$. The one-body energy spectrum is divided into $J$ cells, particles are distributed at random over these, those in the $j$ th cell are regarded as mutually identical, and the simple-minded Boltzmann-Shannon entropy functional

$$
\begin{aligned}
S^{B}= & -k \sum_{j=1}^{J} g_{j}\left\{f_{j}^{B} \ln f_{j}^{B}-f_{j}^{B}\right\} \\
& +k \alpha\left(\sum_{j=1}^{J} g_{j} f_{j}^{B}-N\right)-k \beta\left(\sum_{j=1}^{J} g_{j} f_{j}^{B} \varepsilon_{j}-E\right)
\end{aligned}
$$

is set up. Here $k$ is the Boltzmann constant, $g_{i}$ the cell degeneracy, $N$ the total number of particles, $E$ the total energy, and $\alpha$ and $\beta$ are Lagrange multipliers (see Appendix A for precise definitions of various symbols). Next, one maximizes $S^{B}$ with respect to $f_{j}^{B}, \alpha$, and $\beta$ to arrive at the $\mathrm{MB}$ solution

$$
f^{B}=e^{\alpha-\beta \varepsilon} ; \quad \nu^{B}=g f^{B}: \quad \varepsilon_{0} \leq \varepsilon \leq \infty,
$$

where the index $j$ has been dropped in the quasicontinuum limit, $\varepsilon_{0}$ is the ground level, and the upper end of the simpleparticle energy spectrum has been extended to $+\infty$ both for confining as well as nonconfining potentials $W(r)$. Although (2) has been widely applied $[1,2]$ to gases/liquids kept in the laboratory, yet its application to open astronomical systems leads to the following serious conceptual puzzles.

\subsubsection{Entropy}

In the case of gravitational systems, one always looks for the local (not global) maxima of the entropy functional. The MB solution (2) does this job exactly for the BoltzmannShannon entropy defined by (1), but only approximately for the more sophisticated combinatorial entropy defined by (10) later. It will be shown in Section 3 that the tail of the $M B$ solution becomes illogical in the energy cells of small occupation numbers.

\subsubsection{Density}

If (2) is inserted back into the general expression (A.7) of Appendix A for the mass density $\rho(r)$, one obtains the famous Boltzmann barometric formula

$$
\rho^{B}(r) \propto \exp \left\{-\frac{W(r)}{k T}\right\}: r_{0} \leq r \leq \infty .
$$

The attractive short-distance behaviour of $W(r)$ cannot pose a real problem because the size $r_{0}$ of the quantum ground level $\varepsilon_{0}$ is finite [6]. But the long-distance behaviour of $W(r)$ is problematical as regards astrophysics in $D=3$ dimensions. Indeed, for a dilute gaseous star [2, page 114] without the Poisson equation constraint, one finds asymptotically

$$
W(r) \underset{r \rightarrow \infty}{\sim}-0 ; \quad \rho^{B}(r) \underset{r \rightarrow \infty}{\sim} \text { constant : Gaseous. }
$$

Also, for the isothermal Emden sphere $[3,8]$ subject to the Poisson equation constraint, one knows that

$$
W(r) \underset{r \rightarrow \infty}{\sim} \ln \left(\frac{r^{2}}{a^{2}}\right) ; \quad \rho^{B}(r) \underset{r \rightarrow \infty}{\sim} r^{-2}: \text { Emden }
$$

with $a$ being the isothermal length scale. Clearly, as $r \rightarrow \infty$, the nil/slow decrease of $\rho^{B}$ in (4) and (5) and the logarithmic increase of $W$ in (5) are unphysical.

\subsubsection{Radius}

From the MB density (3), one computes the mean size $\langle r\rangle^{B}$ of the system via

$$
\langle r\rangle^{B}=\frac{\int_{0}^{\infty} \partial r r^{3} \rho^{B}}{\int_{0}^{\infty} \partial r r^{2} \rho^{B}}=\infty
$$

which diverges both for gaseous stars (4) and Emden spheres (5).

\subsubsection{Mass}

The total mass of the MB system is calculated from

$$
M^{B}=4 \pi \int_{0}^{\infty} \partial r r^{2} \rho^{B}(r)=\infty
$$

which also diverges for the two cases mentioned above. Thus, in the Boltzmann-Shannon view, the most likely state of an isotropic stellar system has infinite mass.

\subsubsection{Evaporation}

Since all regions of the phase space up to $r=\infty$ are allowed an open MB system, for example, the dilute gaseous star goes on evaporating with time, producing as a thereby a net outgoing flux $\phi^{B}$ of particles [2, page 114] at every positive thermodynamic temperature $T$ :

$$
\varphi^{B}>0 \text {. }
$$

Of course, the isothermal sphere can be stable against evaporation [9] but its mean field growing like $\ln \left(r^{2} / a^{2}\right)$ up to $r=\infty$ is unphysical. 


\section{King-Like Lowered Isothermal Models}

In the conventional literature, the above difficulties are usually circumvented by enclosing the system within a hypothetical box of some radius $R_{\mathrm{box}}$ [7], or by modifying the original distribution heuristically into non-Maxwellian form $f^{\text {Low }}$ such as

$$
\begin{aligned}
f^{\text {Low }} & \propto \exp \left(-\frac{\varepsilon}{k T}\right)-1, \\
& \propto \exp \left(-\frac{\varepsilon}{k T}\right)+\frac{\varepsilon}{k T}-1,
\end{aligned}
$$

and so forth, holding in the range $\varepsilon<0$ and vanishing elsewhere [4, 8, 16-19]. In particular, King [4] and Wilson [19] appealed to the tidal force field of the galaxy for physically setting its outer boundary and assumed the velocity distribution of stars to be cut off at the local escape velocity. Lowered isothermal prescriptions such as (9) are often employed by astronomers to fit data.

\section{Physical Motivation for $f^{\text {Low }}$}

If one takes a stellar cluster in an original Liouville collisionless state then the cluster will start evolving in space-time through trajectory mixing and stellar encounters which are most frequent in the core region. Mathematically, the complicated dynamics of such a nonequilibrium system is governed by the coupled Fokker-Planck and Poisson equations [17]. Physically, this evolution will involve momentum/mass/heat flow, tide generation, and entropy production. At equilibrium, the macroscopic flows will stop, tides will stabilize, and the entropy would become maximum. Naturally, von Hoerner [20] and King [14] realized that a finite boundary to the star cluster is set up by the tidal force of the galaxy, that is, the cutoff tail in the essentially classical stellar systems can be ascribed to the physical outcome of the boundary conditions and/or constraints (independent of the Plank constant).

\section{Our Most Probable Distribution (MPD)}

\subsection{Preliminaries}

We adopt the view that the above-mentioned King models can be refined further by utilizing the following facts. (i) At equilibrium, the entropy of a multiparticle thermal system should become a (local) maximum. Of course, the Boltzmann-Shannon definition of $S^{B}$ in (1) will not serve the purpose due to the difficulties of the Maxwellian; we shall show in (11) and (12) below that a more suitable candidate is the so-called combinatorial entropy $S$ that counts the number of microstates in energy cells corresponding to specified total particle number $N$ and total energy $E$. (ii) The resulting most probable distribution $f$ should develop a tail which is automatically truncated at a finite energy $\varepsilon_{K}$. This is because a star moving in the mean potential field $W(r)$ will have a farthest turning at distance $r_{K}$ satisfying $W\left(r_{K}\right)=$ $\varepsilon_{K}$, where $r_{K}$ may now be identified with the classical King radius of the galaxy. (iii) By Bohr's correspondence principle, classical motion is the limiting case of quantum motion in states of very large quantum numbers. The cutoff quantum number $K$ and cutoff energy $\varepsilon_{K}$ should be determinable from the variational constraint equations of our MPD theory provided that $h$ is brought into the picture explicitly. (iv) Our MPD solution for $f$ should be able to provide a theoretical justification (or better characterization) of the lowered isothermal Maxwellian models (9). Now we shall demonstrate how such a task is accomplished in practice.

\section{Gibbs Combinatorial Entropy}

We follow the basic theme of Huang [1, page 182], and a preliminary investigation by Menon and Agrawal [12] as well as by Menon et al. [13]. The single-particle spectrum is divided into $J$ cells into which the particles are distributed at random such that the $j$ th cell has central energy $\varepsilon_{j}$, width $\Delta_{j}$, degeneracy $g_{j}$, occupation number $v_{j}$, and occupation probability per state $f_{j}=v_{j} / g_{j}$ defined by Appendix A. Next, treating the particle in the $j$ th cell as indistinguishable, a Gibbs combinatorial entropy functional $S$ is constructed via

$$
S=k \ln \left\{\prod_{j=1}^{J} \frac{g_{j}^{v_{j}}}{v_{j} !}\right\} .
$$

\section{Gamma Function Form}

We deliberately rewrite (10) in the equivalent form

$$
S=k \sum_{j=1}^{J}\left\{\nu_{j} \ln g_{j}-\ln \Gamma\left(\nu_{j}+1\right)\right\} .
$$

The replacement of factorials by gammas has several algebraic advantages. (i) The equality $v_{j} !=\Gamma\left(\nu_{j}+1\right)$ is exact at the integer values $v_{j}=0,1,2,3, \ldots, \infty$. (ii) The asymptotic behaviour, namely, $(2 \pi)^{1 / 2} v^{\nu+1 / 2} e^{-\nu}$ of both $\nu$ ! and $\Gamma(\nu+1)$ are the same as $v \rightarrow \infty$. (iii) Hence, by a theorem due to Carlson [21, 22], $\Gamma(\nu+1)$ provides the most economical, essentially unique continuation of $\nu$ ! to all continuous values throughout the range $0 \leq v \leq \infty$. (iv) While setting up the variational conditions, later we shall need to replace the derivative $\partial \ln \Gamma\left(v_{j}+1\right) / \partial v_{j}$ evaluated at integer values by the digamma function $\psi(v+1)$ [23] computed at general continuous values. This problem of integer programming is handled in Appendix B by using an efficient finite-difference package for all natural numbers up to 4. (v) Appendix B also shows that the numerical differentiation of $\ln \Gamma(\nu+1)$ can be readily done even at small values $v=0.2,0.4$, and so forth, giving results in good agreement with $\psi(v+1)$.

\section{Exact Variational Conditions}

Next, we consider the following objective functional to be maximized:

$$
S^{*}=S+k \alpha\left(\sum_{j} v_{j}-N\right)-k \beta\left(\sum_{j} v_{j} \varepsilon_{j}-E\right),
$$


where $\alpha$ and $\beta$ are unknown Lagrange multipliers. Equating to zero, the partial derivatives $\partial S^{*} / \partial v_{j}, \partial S^{*} / \partial \alpha$, and $\partial S^{*} / \partial \beta$ lead to the following set of exact variational conditions still using the discrete index $j[12]$ :

$$
\begin{gathered}
\psi\left(\nu_{j}+1\right)=\ln g_{j}+\alpha-\beta \varepsilon_{j}: \quad 1 \leq j \leq J, \\
\sum_{j} v_{j}=N ; \quad \sum_{j} v_{j} \varepsilon_{j}=E .
\end{gathered}
$$

\section{Comments}

Without making any assumption concerning the largeness or smallness of $\nu_{j}$, we can rewrite (13a) in the compact form

$$
\psi\left(\nu_{j}+1\right) \underset{\text { exact }}{=} \ln v_{j}^{B} ; \quad v_{j}^{B} \underset{\mathrm{def}}{\equiv} g_{j} e^{\alpha-\beta \varepsilon_{j}},
$$

where the symbol $v_{j}^{B}$ was already encountered earlier in (2). In principle, (14a) can be solved for the desired cell occupation numbers $v_{j}$ in terms of $\alpha, \beta, \varepsilon_{j}$. Thereafter, the Lagrange multipliers $\alpha$ and $\beta$ can be determined from the constraints (13b). Equivalently, the chemical potential $\mu$ and thermodynamic temperature $T$ may be introduced via

$$
k \alpha=\frac{\mu}{T}=\frac{-\partial S^{*}}{\partial N} ; \quad k \beta=\frac{1}{T} \equiv \frac{\partial S^{*}}{\partial E} .
$$

Finally, if the total number $J$ of levels is very large, we are permitted to take the quasilimit (A.4) leading to a continuous distribution for $\nu$ versus $\varepsilon$ by dropping the index $j$ and converting sums into integrals. Let us derive several interesting properties of our most probable distribution (MPD) defined by (13a), (13b) and (14a), (14b) with the suffix $j$ omitted.

\section{Location of the Peak}

Differentiating (14a) with respect to $\varepsilon$, we get

$$
\frac{\partial \psi(\nu+1)}{\partial \nu} \cdot \frac{\partial \nu}{\partial \varepsilon}=\frac{1}{\nu^{B}} \cdot \frac{\partial \nu^{B}}{\partial \varepsilon} .
$$

Clearly, the MPD occupancy $v$ and $M B$ occupancy $v^{B}$ are both peaked at a common energy $\varepsilon_{p}$ which satisfies

$$
\begin{gathered}
\dot{v}\left(\varepsilon_{p}\right)=0 ; \quad \dot{\nu}^{B}\left(\varepsilon_{p}\right)=0, \\
\frac{\dot{g}\left(\varepsilon_{p}\right)}{g\left(\varepsilon_{p}\right)}-\beta=0,
\end{gathered}
$$

where the dot stands for derivative with respect to $\varepsilon$. Typical algebraic estimates of $\varepsilon_{p}$ for the soluble potential models will be reported later in (28).

\section{Large v Region}

In the so-called head region of the continuous distribution, the cells have large occupancy $v \gg 1$ so that the Stirling's approximation $\psi(\nu+1) \approx \ln v+O(1 / v)$ holds in the fundamental equation (14a). Hence the MB solution is roughly retrieved, namely,

$$
\nu \approx v^{B} \gg 1 ; \quad f \approx e^{\alpha-\beta \varepsilon}: \text { Large } \nu,
$$

but it must be violated in the cells where the occupancy becomes comparable to, or less than, unity.

\section{The Tail Region}

On the other extreme lies the tail region of the continuous distribution where the cell occupancy becomes small, that is, $v \ll 1$. Then the digamma function possesses a Taylor expansion

$$
\psi(\nu+1) \underset{\nu \ll 1}{\approx} \psi(1)+\zeta(2) \nu+O\left(\nu^{2}\right): \text { Tail, }
$$

where $\psi(1)=-0.577$ is the negative of Euler's constant and $\zeta(2)=\pi^{2} / 6$ is a Riemann zeta value. Substitution of the expansion (18) into the fundamental equation (13a) leads to the following three surprising yet important observations.

(i) The tail of the distribution intersects the energy axis at a cutoff point $\varepsilon_{K}$ such that

$$
\nu_{K}=0 ; \quad \psi(1)=\ln \nu_{K}^{B} \equiv \ln g_{K}+\alpha-\beta \varepsilon_{K},
$$

where the suffix $K$ refers to energy $\varepsilon_{K}$.

(ii) The said intersection happens linearly because, in its neighbourhood, the occupancy

$$
\nu \approx \frac{\left(\varepsilon_{K}-\varepsilon\right)}{\zeta(2)}\left\{\beta-\frac{\dot{g}_{K}}{g_{K}}\right\}: \text { Tail, }
$$

where $\dot{g}_{K}$ stands for $\partial g / \partial \varepsilon$ evaluated at $\varepsilon_{K}$.

(iii) Extension of the graph of $v$ versus $\varepsilon$ beyond the cutoff point is not allowed because that would tend to make $v$ negative in (13a), that is,

$$
v_{j} \equiv 0 ; \quad f(\varepsilon) \equiv 0: \quad j \geq K, \varepsilon \geq \varepsilon_{K},
$$

implying that the original occupied spectrum (A.2) has shrunk below $J$ or $\varepsilon_{J}$ due to strict entropy maximization under stable equilibrium. (The possibility $K>J, \varepsilon_{K}>\varepsilon_{J}$ would correspond to unstable equilibrium, that is, continuous evaporation of the system.) Schematic plots of $f^{B}$ and $f$ versus $\varepsilon$ are shown in Figure 1. Typical algebraic estimates of the cutoff energy $\varepsilon_{K}$ and quantum number $K$ for the solvable models will be reported later in (28).

\section{Compact Solution for $\nu(\varepsilon)$}

Our equation (14a) is a transcendental equation in $v$ and its precise analytical solution in closed form is not known. Fortunately, there exists an ansatz

$$
\psi(\nu+1) \underset{\text { ansatz }}{=} \ln \{\nu+\theta\} ; \quad \theta \equiv e^{\psi(1)}=0.562,
$$

which works excellently throughout $0 \leq v \leq \infty$ as shown graphically in Figure 2. Combining (14a) and (22), we obtain 


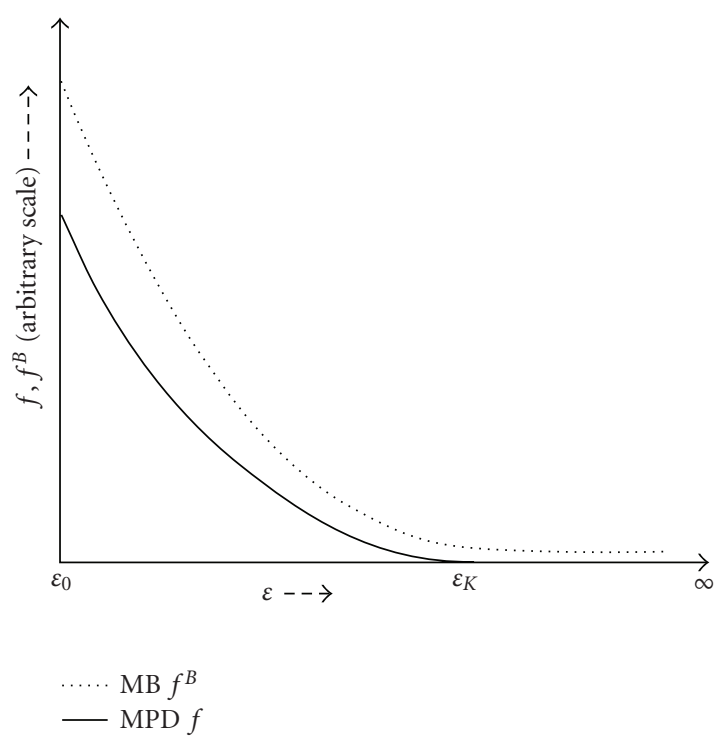

Figure 1: Schematic plots (not to scale) of the distribution functions in the MB approach (cf. (2)) and MPD theory (cf. (23)). (Plot of the occupancy function would have shown a peak in both cases.)

a very compact, quite accurate, MPD solution valid in all energy cells of relevance as

$$
\nu=v^{B}-\theta ; \quad f=f^{B}-\frac{\theta}{g}: \quad \varepsilon_{1} \leq \varepsilon \leq \varepsilon_{K} .
$$

It is interesting to note that if $g$ were replaced by a constant in (23), our MPD solution $f$ would agree with the first line of (9) implying a sort of justification for the lowered isothermal Maxwellian models. Actually, our numerically accurate solution (23) should be regarded as a better characterization since the degeneracy function $g(\varepsilon)$ is strongly energy-dependent.

\section{Compact Number Condition}

Combining the number constraint (13b) with the general solution (23), we can define an effective number $N^{\text {eff }}$ through

$$
\begin{aligned}
N^{\mathrm{eff}} & \equiv N+K \theta=\sum_{j=1}^{K}\left(\nu_{j}+\theta\right)=\sum_{j=1}^{K} v_{j}^{B} \\
& =\int_{\varepsilon_{0}}^{\varepsilon_{K}} \frac{\partial \varepsilon}{\Delta} \cdot g e^{\alpha-\beta \varepsilon}=e^{\alpha} \cdot N^{*} .
\end{aligned}
$$

Here we have employed the quasicontinuum limit (A.4) and introduced

$$
N^{*} \equiv \int_{\varepsilon_{0}}^{\varepsilon_{K}} \partial \varepsilon w(\varepsilon) e^{-\beta \varepsilon} ; \quad e^{\alpha}=\frac{N^{\mathrm{eff}}}{N^{*}} .
$$

This gives a formal expression for the Lagrange multiplier (or reduced chemical potential) $\alpha$ provided that the underlying mean field $W$ or its reduced degeneracy function $w=g / \Delta$ is known.

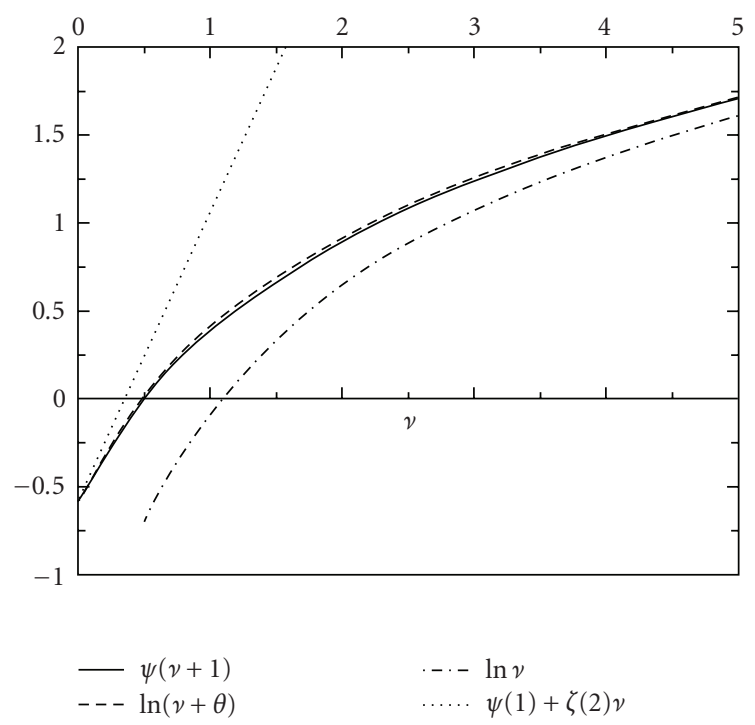

FIGURE 2: The exact digamma function $\psi(\nu+1)$ and three numerical approximations to thesame used in the context of (22), (17), and (18). The values of three important constants are $\psi(1)=$ $-0.577, \theta=e^{\psi(1)}=0.562$, and $\varsigma(2)=\pi^{2} / 6=1.646$.

\section{Compact Cutoff Condition}

Lastly, we convert the cutoff criterion (19) into

$$
\theta \equiv e^{\psi(1)}=\nu_{K}^{B}=g_{K} e^{\alpha-\beta \varepsilon_{K}} .
$$

Eliminating $e^{\alpha}$ with the help of $(24 \mathrm{~b})$, we find

$$
e^{\beta \varepsilon_{K}}=\frac{N^{\mathrm{eff}} g_{K}}{N^{*} \theta}
$$

which yields a formal expression for the cutoff energy $\varepsilon_{K}=\varepsilon(K)$ whose functional dependence can be inverted to specify also the number $K$ of levels. The sharply cutoff tail of (21) will play a crucial role in the cosmological application to be discussed later in Section 4.

\section{Illustration for the (Truncated) Oscillator Well}

The above methodology may be illustrated in the case of the truncated harmonic oscillator potential listed in Table 1:

$$
W(r)=-\left|W_{0}\right|\left\{1-\frac{r^{2}}{R^{2}}\right\} \cdot \operatorname{step}(R-r) .
$$

Before going ahead with the algebra, the following important remarks should be kept in mind. (a) If the well was untruncated, that is, the step function in (26) was absent then all particles would remain truly confined, the Boltzmann mass density (3) would vanish asymptotically, and the MB distribution would not be problematical. (b) However, if the well is truncated by the use of the step function in (26), then the potential vanishes for $r>R$, particles can be ejected into the continuum, the MB distribution becomes problematical, and the MPD philosophy becomes very useful. (c) Near the 
origin the oscillator potential is rather flat, that is, smoothly varying so that it can approximately mimic the realistic mean field in the core region of astronomical galaxies. In sharp contrast, the truncated-linear and Coulomb-like potentials of Table 1 cannot do so since these vary rather quickly as $r \rightarrow 0$. (d) As they stand, the depth $W_{0}$ and range $R$ are only illustrative parameters introduced in (26). However, when we come to cosmological applications in Section 4 (especially the Poisson equation), it will be found that these parameters are directly related to the physical mass $M$ and observed radius $r_{K}$ of the galaxy. We are now ready to apply the MPD program to (26).

\section{The Tilde Nomenclature}

First, we read off the symbols $J, \varepsilon, \Delta, B$, and $g$ from the fourth column of Table 1. Next, for algebraic convenience, the following dimensionless quantities are defined along with the thermal de Broglie wavelength $\lambda$ :

$$
\begin{gathered}
\tilde{J} \equiv \frac{J}{\left|\beta W_{0}\right|^{1 / 2}}=\frac{\left(m\left|W_{0}\right| / 2\right)^{1 / 2} R / \hbar}{\left(\beta\left|W_{0}\right|\right)^{1 / 2}}=\frac{\pi R}{\lambda} \gg 1, \\
\lambda=\pi \hbar\left(\frac{2 \beta}{m}\right)^{1 / 2} ; \quad \widetilde{\varepsilon} \equiv \beta\left(\varepsilon-W_{0}\right)=\frac{j}{\widetilde{J}}, \\
\tilde{\alpha} \equiv \alpha-\beta W_{0}, \quad \widetilde{\varepsilon}_{K} \equiv \beta\left(\varepsilon_{K}-W_{0}\right)=\frac{K}{\widetilde{J}}, \\
j=\tilde{J} \widetilde{\varepsilon}, \quad g=\left(D B \widetilde{J}^{D-1}\right) \widetilde{\mathcal{\varepsilon}}^{D-1}, \\
\tilde{N} \equiv e^{\beta W_{0}} N^{*} \equiv g \int_{0}^{\alpha-\beta \varepsilon}=g e^{\tilde{\alpha}-\tilde{\varepsilon}}, \\
=D B\left(D B j^{D-1}\right) e^{-j / \tilde{J}} \\
\tilde{J}_{K}, \quad \gamma_{K} \equiv \gamma\left(D, \widetilde{\varepsilon}_{K}\right) .
\end{gathered}
$$

A few remarks are in order concerning these definitions. The Planck constant $h$ or thermal de Broglie wavelength $\lambda \equiv h /(2 m k T)^{1 / 2}$ has appeared in the value of the symbol $\tilde{J}$ and the inequality $\lambda / R \ll 1$ is essential for the validity of classical motion (cf. (A.8)). The function $\tilde{\mathcal{\varepsilon}}$ measures the single-particle energy from the ground level in terms of $k T$. The symbols $\tilde{\alpha}$ and $\widetilde{\mathcal{\varepsilon}}_{K}$ may be called the dimensionless chemical potential and dimensionless cutoff energy, respectively, whose fixation using MPD constraints is yet to be done. The integral $\tilde{N}$ will play a crucial role below with $\gamma$ being the incomplete gamma function.

\section{Use of MPD Conditions}

Remembering the tilde quantities, we can readily evaluate the conditions (16), (24b), and (25b). This yields the peak location $\widetilde{\varepsilon}_{p}$, peak height $\nu_{p}^{B}$, dimensionless chemical potential $\tilde{\alpha}$, and dimensionless cutoff energy $\widetilde{\varepsilon}_{K}$ through

$$
\begin{gathered}
\tilde{\varepsilon}_{p}=D-1 \sim 1 ; \quad v_{p}^{B}=g_{p} e^{\tilde{\alpha}-\tilde{\varepsilon}_{p}} \sim \frac{N^{\text {eff }}}{\widetilde{J} \gamma_{K}}, \\
e^{\tilde{\alpha}} \sim \frac{N^{\text {eff }}}{\widetilde{J^{D} \gamma_{K}}} ; \quad \frac{e^{\tilde{\varepsilon}_{K}}}{\tilde{\varepsilon}_{K}^{D-1}} \sim \frac{N^{\text {eff }}}{\widetilde{J} \gamma_{K}} \\
N^{\text {eff }} \equiv N+K \theta ; \quad D>1,
\end{gathered}
$$

where the wavy symbol $\sim$ implies the order of magnitude, and the multiplicative factors of order unity have been suppressed. We still have to show that the formal equations (28) do admit valid, that is, self-consistent MPD solutions under suitable restrictions. For this purpose, we consider below two cases in which the parameter $\widetilde{\varepsilon}_{K}=K / \tilde{J}$ has markedly different behaviours.

Case 1 (well depth large compared to $k$ times temperature). For the truncated oscillator potential (26), we recall the tilde notations (27) and impose the following inequalities:

$$
\begin{gathered}
\tilde{\varepsilon}_{K}=\frac{K}{\widetilde{J}} \gg 1 ; \quad\left|\beta W_{0}\right|=\frac{\left|W_{0}\right|}{k T} \gg 1, \\
\tilde{J} \sim \frac{R}{\lambda} \gg 1 ; \quad \tilde{J} \ll N^{\mathrm{eff}} \ll \tilde{J}^{D}, \\
J=\left(\beta\left|W_{0}\right|\right)^{1 / 2} \tilde{J}>K \gg \tilde{J} \gg 1 .
\end{gathered}
$$

The physical meaning of these restrictions is as follows. The inequalities $J \gg 1, K \gg 1, \tilde{J} \gg 1$ guarantee the validity of classical dynamics in states of large quantum numbers, the condition $J>K$ ensures that the $K$ th level lies below the ionization threshold for stable MPD, the assumption $\widetilde{\varepsilon}_{K} \gg 1$ in Case 1 implies that the actual cutoff energy $\varepsilon_{K}$ is several $k T$ above the ground level, $\left|\beta W_{0}\right| \gg 1$ means that the well depth is large compared to $k T$, the condition $\tilde{J} \ll N^{\text {eff }}$ implies that the effective number $N^{\text {eff }} / \tilde{J}$ of particles grossly counted per cell is much more than unity, and $N^{\text {eff }} \ll \widetilde{J}^{D}$ means that the system is dilute or nondegenerate (because the packing fraction $N^{\text {eff }} / \widetilde{J}^{D} \sim N^{\text {eff }} \lambda^{D} / R^{D} \ll 1$, that is, the average number of particles contained inside a $D$-dimensional sphere of radius $\lambda$ is small compared to unity). Then the incomplete gamma function $\gamma_{K} \rightarrow \Gamma(D)$ and consistent handling of (28) leads to the estimates

$$
\begin{gathered}
\tilde{\varepsilon}_{p} \sim 1 ; \quad v_{p}^{B} \sim \frac{N^{\text {eff }}}{\widetilde{J}} \gg 1, \\
\tilde{\alpha} \sim \ln \left(\frac{N^{\text {eff }}}{\widetilde{J}^{D}}\right) ; \quad \tilde{\varepsilon}_{K} \sim \ln \left(\frac{N^{\text {eff }}}{\widetilde{J}}\right), \\
K \sim \tilde{J}_{\tilde{\varepsilon}_{K}} \sim\left(\frac{R}{\lambda}\right) \ln \left(\frac{N^{\text {eff }} \lambda}{R}\right) .
\end{gathered}
$$

The present case should apply to usual gases/liquids contained in the laboratory and we have independently verified that the functional forms of (29) and (30) are very rugged, that is, they hold for all the soluble models reported in Table 1. 
Table 1: Properties of one-body semiclassical spectrum labeled by the integer $j$ for four solvable potentials $W(r)$ in $D$ dimensions. The well-depth $W_{0} \equiv-|W(0)|$ and the range $R$ of the rectangular, linear, and oscillator wells are made finite using the unit step function. The Coulomb well is left unrestricted over all $r$. For other notations, see Appendix A. (We do not tabulate the rigid box potential model which has the upper end of the energy spectrum at $\varepsilon_{J}=E-(N-1) W_{0} \approx+\infty$. Such a model is of little interest in cosmology In usual physical applications, one puts $D=3$.)

\begin{tabular}{|c|c|c|c|c|}
\hline Potential & Rectangular & Linear & Oscillator & Coulomb \\
\hline Form $W(r)=$ & $W_{0} \operatorname{step}(R-r)$ & $W_{0}\left\{1-\frac{r}{R}\right\} \cdot \operatorname{step}(R-r)$ & $W_{0}\left\{1-\frac{r^{2}}{R^{2}}\right\} \cdot \operatorname{step}(R-r)$ & $\frac{C}{r}=\frac{\varepsilon_{1} r_{1}}{r}: 0 \leq r \leq \infty$ \\
\hline Highest label $J=$ & $\frac{\left(8 m\left|W_{0}\right|\right)^{1 / 2} R}{\pi \hbar}$ & $\frac{4}{3} \cdot \frac{\left(2 m\left|W_{0}\right|\right)^{1 / 2} R}{\pi \hbar}$ & $\left(\frac{m\left|W_{0}\right|}{2}\right)^{1 / 2} \frac{R}{\hbar}$ & $\infty$ \\
\hline Ionization threshold $\varepsilon_{J}=$ & 0 & 0 & 0 & $0=\varepsilon_{\infty}$ \\
\hline Spectrum $\varepsilon_{j}=$ & $W_{0}\left\{1-\left(\frac{j}{J}\right)^{2}\right\}$ & $W_{0}\left\{1-\left(\frac{j}{J}\right)^{2 / 3}\right\}$ & $W_{0}\left\{1-\left(\frac{j}{J}\right)\right\}$ & $\frac{-m C^{2}}{2 \hbar^{2} j^{2}}=\frac{\varepsilon_{1}}{j^{2}}$ \\
\hline Turning point $r_{j}=$ & $R$ & $\left(\frac{j}{J}\right)^{2 / 3} R$ & $\left(\frac{j}{J}\right)^{1 / 2} R$ & $\frac{2 \hbar^{2} j^{2}}{m|C|}=r_{1} j^{2}$ \\
\hline Separation $\Delta=$ & $\frac{2\left|W_{0}\right| j}{J^{2}}$ & $\frac{2}{3} \cdot \frac{\left|W_{0}\right| j^{-1 / 3}}{J^{2 / 3}}$ & $\frac{\left|W_{0}\right|}{J}$ & $\frac{2\left|\varepsilon_{1}\right|}{j^{3}}$ \\
\hline Geometrical coefficient $B=$ & $\frac{\pi^{D}}{2^{2 D-2} D^{2} \Gamma^{2}(D / 2)}$ & $\frac{(3 \pi / 4)^{D} \Gamma(D)}{2^{D-1} \Gamma(D / 2) \Gamma(3 D / 2+1)}$ & $\frac{1}{\Gamma(D+1)}$ & $\frac{1}{D \Gamma(D / 2+1 / 2)}$ \\
\hline Accumulated states $I_{j}$ & $B j^{D}$ & $B j^{D}$ & $B j^{D}$ & $B j^{D}$ \\
\hline Level degeneracy $g_{j}=$ & $D B j^{D-1}$ & $D B j^{D-1}$ & $D B j^{D-1}$ & $D B j^{D-1}$ \\
\hline
\end{tabular}

Case 2 (well depth comparable to $k$ times temperature). Again we recall the tilde notations (27) and impose the orders of magnitude

$$
\widetilde{\varepsilon}_{K}=\frac{K}{\widetilde{J}} \sim 1 ; \quad\left|\frac{W_{0}}{k T}\right| \sim 1
$$

Then the incomplete gamma function $\gamma_{K} \sim 1$ and (28) are found to admit the self-consistent estimates

$$
\begin{gathered}
\widetilde{\varepsilon}_{p} \sim 1 ; \quad v_{p}^{B} \sim \frac{N^{\mathrm{eff}}}{\tilde{J}} \sim 1, \\
N^{\mathrm{eff}} \sim N \sim K \sim \tilde{J} \sim \frac{R}{\lambda} \gg 1, \\
\tilde{\alpha} \sim \ln \left(\frac{1}{\tilde{J}^{D-1}}\right) ; \quad \tilde{\varepsilon}_{K} \sim \ln \left(\frac{N^{\mathrm{eff}}}{\widetilde{J}}\right) \sim 1 .
\end{gathered}
$$

The physics of (31) and (32) is as follows. The statement $\left|W_{0} / k T\right| \sim 1$ applies to gravitational systems obeying virialization, $N \sim K$ tells that the total number of particles is of the same order as the number of MPD cells, and $\nu_{p}^{B} \sim 1$ signifies that the cell occupancies have become comparable to unity with $\hbar$ again playing a role through the symbol $\tilde{J}$. The present case should correspond to open astronomical systems and the ruggedness of the results (32) can be verified also for the other solvable models in Table 1.

\section{Conceptual Application of MPD to Cosmology}

We are now ready to resolve the conceptual difficulties of the MB distribution mentioned already in Section 2 by employing the MPD solution obtained in Section 3.

\subsection{Entropy}

The Boltzmann-Shannon entropy $S^{B}$ of (1) is simpleminded, its maximization leads to the MB solution $\nu^{B}$ in (2) with untruncated tail, and its generalization to quantum statistics is difficult. In sharp contrast, the combinatorial entropy $S$ of (11) is sophisticated, its maximization leads to our MPD solution $v$ in (23) with a truncated tail, and its generalization to quantum statistics as straightforward.

\subsection{Density}

If the MPD information (21) is inserted back into the general expression (A.7) for the local mass density $\rho$, based on the transformation $\sigma=\varepsilon-W(r)$, we obtain

$$
\rho(r) \propto \int_{0}^{\sigma_{K}} \partial \sigma \sigma^{D / 2-1} f(\varepsilon) ; \quad \sigma_{K} \equiv \varepsilon_{K}-W(r)
$$

where $\rho$ surprisingly vanishes if $W(r)$ equals $\varepsilon_{K}$. This is explained by remembering that since no particle in MPD is allowed to have an energy more than $\varepsilon_{K}$, there exists a largest 
classical turning point at $r_{K}$ beyond which the density must become zero identically, that is,

$$
W\left(r_{K}\right)=\varepsilon_{K} ; \quad \rho(r) \equiv 0 \quad \text { for } r \geq r_{K},
$$

in sharp contrast to the MB density profiles (3)-(5). We can also find the rate at which $\rho(r)$ approaches zero as $r$ tends to $r_{K}$. However, (20) has already told us that $f(\varepsilon) \propto \varepsilon_{K}-$ $\varepsilon \propto \sigma_{K}-\sigma$ in the tail region. Hence, (33) yields the leading behaviour

$$
\rho(r) \underset{\sigma_{K} \rightarrow 0}{\propto} \int_{0}^{\sigma_{K}} \partial \sigma \sigma^{D / 2-1} \cdot \sigma \underset{W(r) \rightarrow \varepsilon_{K}}{\propto}\left\{W\left(r_{K}\right)-W(r)\right\}^{D / 2+1} .
$$

Since in a "good" MPD solution $\varepsilon_{K}$ and $r_{K}$ are finite, our result (35) tells that the mass density obeys a $\left(r_{K}-r\right)^{D / 2+1}$ law near the edge of the system.

\subsection{Radius}

Clearly, the distance $r_{K}$ in (34) is the upper bound on the size of our galactic system and, for binding, we must have $r_{K}<r_{J}$ with $r_{J}$ being the turning point just before the continuum starts. (In the soluble models of Table 1 , this $r_{J}$ was called $R)$. Since the density $\rho$ vanishes beyond $r_{K}$ the MPD integral defining the average size $\langle r\rangle$ will also converge, that is,

$$
\langle r\rangle<\infty
$$

in sharp contrast to the MB mean radius (6).

\subsection{Mass}

By the same token, the MPD integral defining the total mass of the stellar system also exists, that is,

$$
M<\infty,
$$

in sharp contrast to the MB mass (7).

\subsection{Nonevaporation}

As is well known if an attractive mean field $W(r)$ vanishes asymptotically, then the energy $\varepsilon=0$ is called the ionization threshold. Hence, our galaxy will be stable against evaporation if the MPD cutoff energy $\varepsilon_{K}$ happens to be negative at the given thermodynamic temperature $T$. Consequently, for $\varepsilon_{K}<0$, there is no net outgoing particle flux, that is,

$$
\varphi=0
$$

in sharp contrast to the MB result (8).

\section{Comment}

Of course, a galaxy which is observed experimentally to evaporate is not in true equilibrium. Then simplifying restrictions like (31) may not hold, that is, the cutoff conditions (19) and (32) will admit a positive root for $\varepsilon_{K}$.

\section{Poisson Equation Implications}

So far in our treatment, the detailed algebraic form of the mean field $W(r)$ was not required explicitly for selfgravitating systems. Actually this is a tough problem theoretically/numerically because one must solve the coupled equations for the distribution function $f$ and mass density $\rho$ in accordance with the Poisson equation in 3 dimensions

$$
\nabla^{2} W \equiv \frac{1}{r^{2}} \frac{\partial}{\partial r}\left(r^{2} \frac{\partial W}{\partial r}\right)=4 \pi G m \rho ; \quad D=3,
$$

where $G$ is the gravitational constant. Our limited aim in the present paper will, however, be served by noting the following features.

\subsection{Features}

(a) Since the density $\rho(r)$ is sharply cutoff at $r_{K}$, by Gauss theorem, the exact potential energy and force at exterior points become

$$
W(r)=\frac{-G M m}{r} ; \quad F(r)=\frac{-G M m}{r^{2}}: \quad r \geq r_{K} .
$$

(b) At the edge $r_{K}$ itself, the potential energy becomes equal to the cutoff energy, namely,

$$
\varepsilon_{K}=W\left(r_{K}\right)=\frac{-G M m}{r_{K}} .
$$

(c) In the interior region, the mean field may get smoothened so as to yield a finite depth

$$
W_{0} \equiv W(0) \sim-k T
$$

by virtue of the gravitational virial theorem.

(d) At interior points, the exact profile of the mean field is not known a priori since it has to be, in general, computed numerically by solving (39). However, for the purposes of illustration, we can represent it by an oscillator form $W(r)=$ $W_{0}\left(1-r^{2} / R^{2}\right)$ if $r \leq r_{K}$ with unknown phenomenological constants $W_{0}$ and $R$. The corresponding interior potential energy and force at the system edge $r_{K}$ then become

$$
W\left(r_{K}\right)=W_{0}\left(1-\frac{r_{K}^{2}}{R^{2}}\right) ; \quad F\left(r_{K}\right)=\frac{2 W_{0} r_{K}}{R^{2}} .
$$

Matching these to the exterior values given by $(40)$ at $r_{K}$, we identify

$$
W_{0}=\frac{3 W\left(r_{K}\right)}{2} ; \quad R=\sqrt{3} r_{K}
$$

Thus, $W_{0}$ is deeper than $W\left(r_{K}\right)$ and $R$ is larger than $r_{K}$ (although orders of magnitude are the same).

\section{Suggested Procedure for Cosmologists}

Suppose a practical astronomical observation has been made on a cluster of $N \sim 10^{5}$ stars. For utilizing our MPD theory with respect to his collected data, the cosmologist should proceed through the following steps. 
Step 1 (characterization parameters). From the observed size $r_{K}$ and the known mass $M$ of the cluster, the MPD cutoff energy $\varepsilon_{K}$ is immediately given by (41) as $\varepsilon_{K}=W\left(r_{K}\right)=$ $-G M m / r_{K}$. Next, the oscillator well-depth $W_{0}$ and the range parameter $R$ for motion inside the cluster are set up from (44) as $W_{0}=3 W\left(r_{K}\right) / 2$ and $R=\sqrt{3} r_{K}$. Next, according to (32) applicable to cosmology, the MPD parameters have the rough orders of magnitude

$$
K \sim N \sim \tilde{J} \sim \frac{R}{\lambda} \sim \frac{\left(m\left|W\left(r_{K}\right)\right|\right)^{1 / 2} r_{K}}{\hbar} \sim\left(\frac{G M m^{2} r_{K}}{\hbar^{2}}\right)^{1 / 2}
$$

upon using the value of $\tilde{J}$ given by the first line of (27) under virialization. Next, the cosmologist may treat (45) as providing a new mass versus radius relationship $M / r_{K} \sim$ $G m^{4} / \hbar^{2}$ for nondegenerate clusters whose experimental status is, however, not yet studied. Finally, for an accurate interlink among all MPD parameters, the astronomer may like to solve the transcendental equations (27) numerically.

Step 2 (MPD density near the edge). Next, the astronomer may look at (35) which gives the leading behaviour of the stellar number density near the cluster's boundary:

$$
n(r) \underset{r \rightarrow r_{K}}{\propto}\left\{W\left(r_{K}\right)-W(r)\right\}^{3 / 2+1} \underset{\mathrm{MPD}}{\propto}\left\{\frac{1}{r}-\frac{1}{r_{K}}\right\}^{5 / 2}
$$

since the mean field $W(r)$ becomes Newtonian near the periphery. This can be cast into more convenient form by defining the variable $x=1 / r$, choosing a normalization point $x_{1}=1 / r_{1}$, and working with the modified function

$$
\hat{n}^{2 / 5} \underset{\text { def }}{\equiv}\left\{\frac{n(r)}{n\left(r_{1}\right)}\right\}^{2 / 5}=\frac{x-x_{K}}{x_{1}-x_{K}}
$$

which becomes unity at $x_{1}$ but vanishes at $x_{K}=1 / r_{K}$. To test the validity of (47), the astronomer may, for example, concentrate on the star counts made on photographs of the cluster M 15 (see Figure 2 of King [14]) taken with the 48-inch Schimdt camera in the Palomer observatory. The results of $\hat{n}^{2 / 5}$ are plotted in Figure 3. Clearly, there is quite good agreement between experimental observation and MPD prediction, although a slight curvature in the data trend may imply the presence of additional weak nonlinear terms on the RHS of (47).

Step 3 (comparison with King density). Next, it is worthwhile to consider the function $\hat{n}^{1 / 2}$ and expand its MPD expression (47) around the matching point $x_{1}$ binomially in the form

$$
\hat{n}^{1 / 2} \underset{\mathrm{MPD}}{=}\{1+\delta\}^{5 / 4} \underset{x \rightarrow x_{1}}{=} 1+\frac{5}{4} \delta+O\left(\delta^{2}\right) ; \quad \delta \equiv \frac{x-x_{1}}{x_{1}-x_{K}} .
$$

Dropping the $\delta^{2}$ term, the cosmologist retrieves the famous formula proposed empirically by King, namely,

$$
\hat{n}^{1 / 2} \underset{\text { King }}{=} \frac{x-x_{t}}{x_{1}-x_{t}} ; \quad x_{t}=\frac{4 x_{K}+x_{1}}{5},
$$

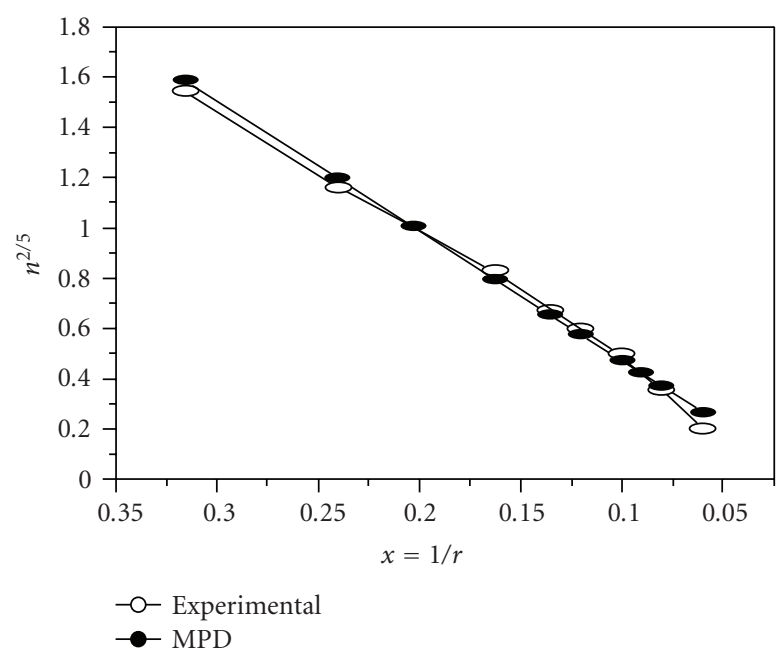

FIGURE 3: Normalized stellar number density raised to 2/5 power, that is, $\hat{n}^{2 / 5} \equiv\left\{n(r) / n\left(r_{1}\right)\right\}^{2 / 5}$ near the edge of the cluster M 15 . The experimental data points are adapted from King [14, Figure 2]. The theoretical MPD prediction is computed from (47) with $x_{1}=$ $0.202, x_{K}=0.008$.

whose square gives the King's profile [14, equation (2)] near the cluster's periphery as

$$
n(r) \underset{\text { King }}{\propto}\left\{\frac{1}{r}-\frac{1}{r_{t}}\right\}^{2} .
$$

It is well known that the phenomenological proposal (49) has been extensively used in the past by astronomers. For example, in context of M15 cluster Figure 4 shows the plot of $\hat{n}^{1 / 2}$ near the cluster's boundary. Clearly, the agreement between experimental observation and King's parametrization is good, ignoring the slight curvature in the data trend. Incidentally, the qualities of fit seen in Figures 3 and 4 are quite comparable implying that, with the present accuracy of measuring $\hat{n}$, it is not possible to say whether MPD formula (47) or King recipe (49) is superior.

Step 4 (complete density profile). Finally, the astronomer may like to have an expression for the number density $n(r)$ valid throughout the range $0 \leq r \leq r_{K}$. In principle, our MPD distribution function $f$ given by (23) yields the formal expression

$$
n(r) \underset{\text { MPD }}{=} \int \frac{\partial^{D} \vec{p}}{h^{D}}\left\{f^{B}-\frac{\theta}{g}\right\}
$$

with the mean potential $W(r)$ being approximately harmonic oscillator in the interior and Newtonian near the edge. Unfortunately, analytical evaluation of the phase space integral (51) is somewhat tedious and will be dealt with in a future communication. However, the cosmologist should note that the integral (51) is the algebraic difference of two terms which is very satisfying because the empirical full density profile written by King [14, equation (14)] also contains a difference of two terms. 
Step 5 (velocity distribution of stars). It is a standard astronomical practice to measure the local radial velocity distributions (along with other properties) of stars in a globular cluster, for example, see the extensive photometric study made by van Loon et al. [15] on the post-mainsequence stars in $\omega$ Centauri (NGC 5139). The cosmologist may ask how well our MPD distribution function $f$ given by (23) fits the observed data. Unfortunately, a straightforward answer to this question is difficult because exact values of the unknown parameters $\alpha$ and $K$ must be obtained numerically from the transcendental conditions (24b) and (25b). We plan to accomplish this task in a future communication.

\section{Concluding Remarks}

The main results of the present work appear in the abstract along with Sections 2-4 and are often emphasized by italics. It is hoped that astronomers will benefit from the algebraic properties of MPD derived in Section 3, its cosmological implications mentioned in Section 4, numerical plots of number density profiles in Figures 3 and 4, and a pointwise comparison between the King model and MPD philosophy made in Table 2. Clearly, both types of theories can be applied to cosmology although our $f$ may be regarded as providing a better characterization from the conceptual viewpoint.

The essence of our cosmological discussion in Section 4 is the following. Suppose that an astronomer makes observations on a (quantum mechanically nondegenerate) cluster having $N$ stars, total mass $M$, and radius $r_{K}$. Then, its MPD solution will be characterized by the cutoff energy $\varepsilon_{K}=-G M m / r_{K}$ and cutoff quantum number $K \sim N$. Before ending the paper, we mention below briefly several important points not discussed explicitly in the earlier sections.

(i) In the mean field description of a multiparticle system, fluctuations arising from short-range pair correlations are usually ignored. The effect of fluctuations is likely to be stronger on the MB solution $f^{B}$ whose tail extends to $\infty$ in (2). Such effect is likely to be weak on the MPD solution $f$ whose tail gets truncated at $\varepsilon_{K}$ in (23).

(ii) One may argue that a sharp radius is also known to arise in the method of self-consistent Hartree fields applied to gravitational systems [17]. We stress, however, that the Hartree method is done through a numerical algorithm because the coupled equation for the mean field and distribution function must be solved iteratively on a computer. Therefore, our analytical maximum-entropy treatment of Section 3 still retains its novelty.

(iii) One may also argue that it is not meaningful to demand thermodynamic equilibration in the peripheral region of the galaxy because, due to low densities, relaxation may remain incomplete there. However, it must be kept in mind that since gravitational forces are of long range, the mechanism of collisionless relaxation [9] still operates. Therefore, our assumption of equilibration even in the tail region may remain justified.

(iv) Next, mention must be made of some recent investigations $[10,11]$ carried out on the question of gravitational galactic clustering, their virialization, and peculiar velocity distribution superposed over the local Hubble flow. These authors start from the $N$ body cosmological canonical partition function $Z_{N}$ in a box of large volume $V$, perform the individual momentum integrals at the outset over the infinite domain $-\infty \leq p \leq \infty$, write the entropy $S$ as the logarithm of a Gibbs integral over the density of states, and minimize the Helmholtz free energy $E-T S$ with respect to the internal energy $E$. Of course, these investigations are very different from our work because we do not need an enclosing box, momentum integrations over infinite domain are never performed, the entropy functional is combinatorial, and maximization is done with respect to the cell occupation numbers.

(v) Next, suppose that one considers a time span long compared to the two-body relaxation time in a globular stellar cluster. One may argue that a star having energy $\varepsilon_{e}=$ -0 (i.e., arbitrarily close to zero but still negative) will go far away and yet come back. Since the corresponding turning point $r_{e}$ may be arbitrarily big, one expects a very small (but not zero) possibility of the star's existence even at a very large radius. This logic apparently contradicts the MPD result (34) which had claimed that there is no density outside a finite distance $r_{K}$.

Actually, the above logic has the following very subtle fault. While doing pure dynamics, it is enough to find trajectories and their turning points $r_{e}$; but while doing statistical mechanics, it is essential also to calculate the density profile $\rho_{e}(r)$ and the related total mass $M_{e}$. Now, in direct analogy with (35) but with $\varepsilon_{e}=-0$, the density profile at large distance and its associated Poisson equation become (in $D=3$ dimensions)

$$
\begin{aligned}
& \rho_{e}(r) \underset{r \rightarrow \infty}{\propto}\left\{-W_{e}(r)\right\}^{5 / 2} \propto \nabla^{2} W_{e}(r) ; \\
& \therefore W_{e}(r) \propto r^{-4 / 3} .
\end{aligned}
$$

This result is physically unacceptable because the gravitational potential due to a finite mass object must fall asymptotically like $1 / r$. Hence a logic based on $\varepsilon_{e}=-0$ will not work. In sharp contrast, if the globular cluster has finite experimental mass $M$, then it can be easily described by our MPD solution (34) characterized by bounded $r_{K}$ and finite $\varepsilon_{K}<-0$.

(vi) Finally, a cosmologist may argue that since real gravitating systems have a mass spectrum of stars, the assumption of particles with the same mass $m$ in MPD may not be justified. We wish to point that some workers have attempted to apply hydrodynamical equations to globular clusters employing a phase space density involving the continuous mass [24] as an extra variable. Some other workers have analyzed phenomenologically the mergence of clusters such as Praesepe [25] employing four mass bins. Although, in principle, a multicomponent combinatorial entropy will now replace (10), yet the corresponding variational conditions (13a) and (13b) will be hard to handle analytically because different chemical potentials and different cutoff energies may have to be assigned to various components present in the 
TABLE 2: Salient features of King-like lowered isothermal models $f^{\text {Low }}$ (cf. (9)) and our MPD distribution $f($ cf. (23)).

\begin{tabular}{|c|c|c|}
\hline Feature & $f^{\text {Low }}$ & $f$ \\
\hline Physical motivation before equilibrium & $\begin{array}{l}\text { As a general stellar cluster evolves tides are } \\
\text { generated }\end{array}$ & $\begin{array}{l}\text { As a general stellar cluster evolves entropy } \\
\text { is produced }\end{array}$ \\
\hline Theoretical basis at equilibrium & Tidal force of the galaxy & Entropy maximization \\
\hline Equation obeyed & $\begin{array}{l}\text { Simple, approximate, and nonunique in } \\
\text { (9) }\end{array}$ & Transcendental, exact, and unique in (23) \\
\hline Role of Planck constant & $\begin{array}{l}\text { Not needed since the treatment is classical } \\
\text { Newtonian. }\end{array}$ & $\begin{array}{l}\text { Needed explicitly to find the cell degener- } \\
\text { acy } g_{j} \text {, the index } \widetilde{J} \text { of }(27) \text {, and the huge } \\
\text { cutoff number } K \text {. }\end{array}$ \\
\hline Upper energy cutoff in the spectrum & Imposed by hand at $\varepsilon=0$ in (9). & $\begin{array}{l}\text { Predicted by theory to occur at } \varepsilon_{K}<0 \text { in } \\
\text { (19). }\end{array}$ \\
\hline Functional form & $\begin{array}{l}\text { For example, } e^{-\beta \varepsilon}-1 \text { in which the sub- } \\
\text { traction term } 1 \text { is constant. }\end{array}$ & $\begin{array}{l}f^{B}-\theta / g \text { in which the subtraction term } \theta / g \\
\text { is strongly energy-dependent. }\end{array}$ \\
\hline Mass and radius of the cluster & Finite & Finite \\
\hline Number density profile near the edge & $n(r) \propto_{\text {King }}\left(1 / r-1 / r_{t}\right)^{2}$ & $n(r) \propto_{\mathrm{MPD}}\left(1 / r-1 / r_{t}\right)^{5 / 2}$ \\
\hline Quality of fit to $n^{1 / 2}$ and $n^{2 / 5}$ & Good in Figure 4 & Good in Figure 3 \\
\hline
\end{tabular}

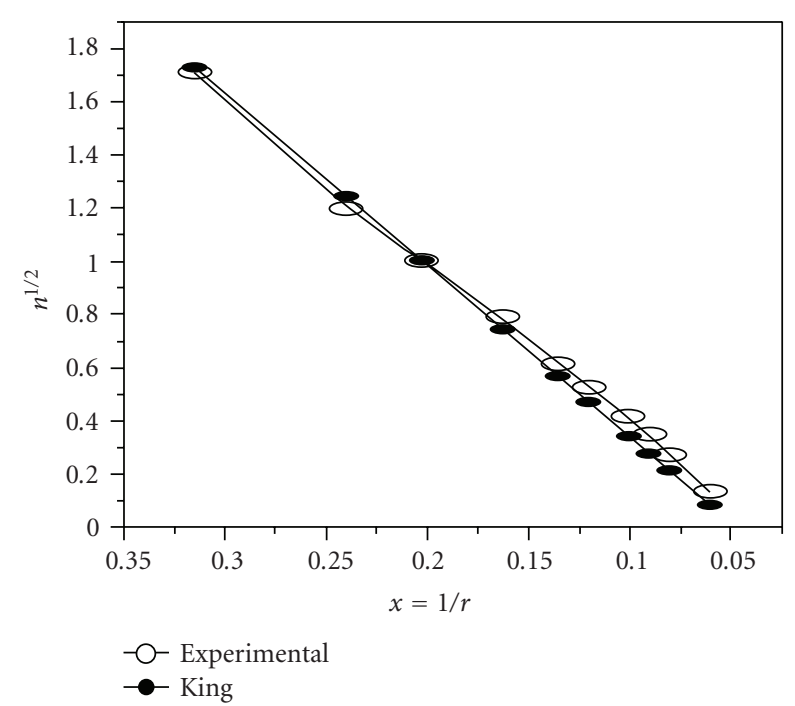

FIGURE 4: Normalized stellar number density raised to $1 / 2$ power, that is, $\hat{n}^{1 / 2}$ near the boundary of the M 15 cluster. The experimental data points are read from King [14, Figure 2]. His model fit is given by (49) with $x_{1}=0.202, x_{t}=0.047$.

system. An easy approximation will be to still use the MPD formalism of Section 3 based on the single particle average mass

$$
m=\frac{\sum_{i} N_{i} m_{i}}{\sum_{i} N_{i}}
$$

where the suffix $i$ runs over different species and there are $N_{i}$ particles of the $i$ th type. This prescription should be reasonable for those clusters where the mass dispersion is small (in units of the solar mass).

\section{Appendices}

\section{A. One-Body Description Recapitulated}

\section{A.1. Preliminaries}

This section will summarize our notations along with several known formulae dealing with the semiclassical singleparticle spectrum/distribution without invoking entropy constraints. Some of these formulae will be used explicitly in Sections 3-5 of the text.

\section{A.2. Assumptions and Notations}

Consider the nonrelativistic localized motion of a particle in $D$ spatial dimensions under the influence of a smooth attractive central field. Classically, the symbols

$$
m, r, p, W(r), \quad F(r)=-W^{\prime}(r), \quad \varepsilon=\frac{p^{2}}{2 m}+W(r)
$$

respectively, denote the mass, distance, absolute momentum, potential energy, applied force, and mechanical energy of this particle. Quantum mechanically invokes the Planck constant $h \equiv 2 \pi \hbar$ and solves the Schrödinger equation for determining the energy spectrum

$$
\varepsilon_{0}<\varepsilon_{1}<\varepsilon_{2}<\cdots<\varepsilon_{J},
$$

where $\varepsilon_{0}$ is the ground level and $\varepsilon_{J}$ the highest bound level supported. Of course, solution of the Schrödinger equation for the exact eigenvalues, eigenfunctions, and their degeneracy is generally tedious.

\section{A.3. Sommerfeld Quantization}

Perhaps the easiest semiclassical link between the descriptions (A.1) and (A.2) is provided by Sommerfeld's criterion 
[26] which says that the phase integral or action variable over a complete oscillation should be an integer multiple of $h$. Then a discrete level $\varepsilon_{j}$ in (A.2) corresponds to the classical turning point $r_{j}$, local momentum variable $p$, principal quantum number $j$, and level spacing $\Delta_{j}$ given by

$$
\begin{gathered}
W\left(r_{j}\right)=\varepsilon_{j} ; \quad p=\left\{2 m\left(\varepsilon_{j}-W(r)\right\}^{1 / 2},\right. \\
j=\frac{2}{\pi \hbar} \int_{0}^{r_{j}} \partial r, \quad p=0,1,2, \ldots, \\
\frac{1}{\Delta_{j}}=\frac{\partial j}{\partial \varepsilon_{j}}=\frac{2 m}{\pi \hbar} \int_{0}^{r_{j}} \frac{\partial r}{p} .
\end{gathered}
$$

Since the presence of zero point energy is of little consequence here, hence the more sophisticated WKB quantization [27] will not be needed for our purpose. Also, if the number $J$ of supported levels is very large compared to unity, then the quasicontinuum limit can be taken by writing

$$
\begin{gathered}
J \gg 1 ; \quad 0 \leq j \leq J ; \quad \varepsilon=\varepsilon_{j}, \\
\Delta=\Delta_{j} ; \quad \sum_{j=0}^{J}=\int_{\varepsilon_{0}}^{\varepsilon_{j}} \frac{\partial \varepsilon}{\Delta} .
\end{gathered}
$$

\section{Gibbs' Prescription}

Further information is obtained by imagining a spherical region of range $R$ and remembering that several quantum states of different orbital angular momenta and magnetic projections may be nearly degenerate at a given energy level. Then, the $D$-dimensional solid angle $\Omega$, total volume $V$ of the region, useful geometrical factor $A$, Gibbs' phase space element $\partial \Xi$, accumulated number $I_{j}$ of quantum states below $\varepsilon_{j}$, local number $w_{j}$ of states per unit energy interval, and the degeneracy $g_{j}$ of the $j$ th level itself are read off from

$$
\begin{gathered}
\Omega=\frac{2 \pi^{D / 2}}{\Gamma}\left(\frac{D}{2}\right) ; \quad V=\frac{\Omega R^{D}}{D}, \\
A=\frac{\Omega^{2}}{h^{D}}=\left\{2^{D-2} \hbar^{D} \Gamma^{2}\left(\frac{D}{2}\right)\right\}^{-1}, \\
\partial \Xi=\frac{\partial^{D} \vec{x} \partial^{D} \vec{p}}{h^{D}}, \\
I_{j}=I(\varepsilon)=\int \partial \Xi \operatorname{step}(\varepsilon-H)=A D^{-1} \int_{0}^{r_{j}} \partial r r^{D-1} p^{D}, \\
w_{j}=w(\varepsilon)=\frac{\partial I}{\partial \varepsilon}=\int \partial \Xi \delta(\varepsilon-H)=m A \int_{0}^{r_{j}} \partial r r^{D-1} p^{D-2}, \\
g_{j}=g(\varepsilon)=\frac{\partial I}{\partial j}=w \Delta .
\end{gathered}
$$

Here $H$ is the single-particle Hamiltonian, the quasicontinuum limit (A.4) is understood, $\Gamma$ is the gamma function, step the unit step function, and $\delta$ the delta function.

\section{Solvable Potential Models}

The methodology described by (A.3)-(A.5) is best illustrated in the case of 4 soluble models, namely, the rectangular, truncated linear, truncated harmonic oscillator, and Coulomb wells. The results are summarized in Table 1 and the following features are worth noticing.

(i) In the case of the rectangular, linear, and oscillator wells, the range $R$ represents the distance beyond which the particle goes into the continuum. By the same token, the highest level $J$ is fixed through the requirement that $\varepsilon_{J}=0$.

(ii) For the Coulomb well, however, since the bound orbits can have any size, one sets $R=\infty$. By the same token, the ionization threshold appears at $J=$ $\infty, \varepsilon_{J}=0$.

(iii) In every model of Table 1 , the semiclassical energy $\varepsilon$ increases monotonically with the principal quantum number $j$, but the trend of the level spacing $\Delta$ is not uniform.

(iv) In every soluble model, the level degeneracy $g_{j}=$ $D B j^{D-1}$, where the geometrical factor $B$ is of order unity. Hence it is reasonable to expect that, for a more general attractive central field in $D=3$ dimensions, $g \propto j^{2}$ at least for large $j$.

(v) Table 1 does not explicitly treat the infinite rectangular well, that is, rigid box in which particles of any momentum would remain confined. Then, the highest kinematically allowed level would have $\varepsilon_{J}=$ $E-(N-1) \varepsilon_{0}$ using the many-body notation of (A.6) below. Of course, the rigid box model is irrelevant in cosmology.

\section{Multiparticle, Statistical System}

In the present paper, we shall not consider pure Bose/Fermi many-body systems where the strict quantum mechanical identity of all particles is crucial. Ours is the so-called Boltzmann system where the one-body spectrum is obtained from the Schrödinger equation/semiclassical quantization but strict identity among all particles is not imposed except within the same energy cell. The mean field $W(r)$ of (A.1) may be either externally applied or internally generated. Assuming spherical symmetry, independent-particle motion, and ignoring short-range pair correlations, we let the symbols

$$
\begin{gathered}
N, E, n=\frac{N}{V}, T, k, \\
\beta=\frac{1}{k T}, \quad \lambda=\frac{h}{(2 m k T)^{1 / 2}}=\pi \hbar\left(\frac{2 \beta}{m}\right)^{1 / 2},
\end{gathered}
$$

respectively, denote the specified number of particles, total energy, global average number density, global mean thermodynamic temperature, Boltzmann constant, inverse temperature parameter, and thermal de Broglie wavelength. The one-body phase space may be imagined to be composed 
of the differential elements $\partial \Xi$ (cf. (A.5)) or of $J$ energy cells of successive widths $\Delta_{j}$ which are arranged in the sequence (A.2). Then a useful transformation $\sigma$, single-particle energy $\varepsilon$, one-body distribution function $f_{j}$, cell occupancy $v_{j}$, local number density $n(r)$, local mass density $\rho(r)$, total number $N$, and total energy $E$ are read off from

$$
\begin{aligned}
\sigma & =\frac{p^{2}}{2 m} \geq 0 ; \quad \varepsilon=\sigma+W(r), \\
f_{j} & =f(\varepsilon) ; \quad \nu_{j}=g_{j} f_{j}=w f \Delta, \\
n(r) & =\frac{\rho(r)}{m}=\int \frac{\partial^{D} \vec{p} f}{h^{D}} \\
& =\frac{1}{\Gamma(D / 2)}\left\{\frac{m}{2 \pi \hbar^{2}}\right\}^{D / 2} \int_{0}^{\varepsilon_{J}-W(r)} \partial \sigma \sigma^{D / 2-1} f, \\
N & =\sum_{\nu=0}^{J} \nu_{j}=\int \partial \Xi f=\int_{\varepsilon_{0}}^{\varepsilon_{J}} \partial \varepsilon w f, \\
E & =\sum_{j=0}^{J} \nu_{j} \varepsilon_{j}=\int \partial \Xi f \cdot \varepsilon=\int_{\varepsilon_{0}}^{\varepsilon_{T}} \partial \varepsilon w f \cdot \varepsilon .
\end{aligned}
$$

Two crucial comments are in order at this stage. (i) The functional form of $f(\varepsilon)$ is left unspecified at the moment. (ii) Convincing justifications are still needed for retaining $h \equiv 2 \pi \hbar$ in our mechanical as well as statistical expressions (A.3)-(A.7) especially when application to classical galaxies of enormous sizes is being envisaged.

\section{Importance of Planck Constant}

(a) By Bohr's correspondence principle, the motion of a quantum Schrödinger/Sommerfeld particle tends to become classical in the states of large principal quantum numbers. In the notation of (A.4), this requires $J \gg 1$ where $J$ of Table 1 contains $\hbar$ explicitly. (b) Strict Bose/Fermi statistical systems tend to obey classical statistics at low density and high temperature if $n \lambda^{D} \ll 1$ in the notation of (A.6). This requires that the linear size of the system be large compared to the thermal de Broglie wavelength, that is, $R \gg \lambda$. Hence the $\hbar$-dependent dual inequalities

$$
J \gg 1 ; \quad R \gg \lambda
$$

tell very precisely when a multiparticle system can be called "classical." Such a precision would be lacking if $\hbar$ were dropped at the outset in cosmological applications. (c) While the Sommerfeld quantum number $j$ in (A.3) is very suitable for labelling the distinct energy levels, the Gibbs degeneracy $g$ (derived from the phase-space element $\partial^{D} \vec{x} \partial^{D} \vec{p} / h^{D}$ ) in Table 1 is equally convenient to count the precise number of states in any cell. (d) The precise knowledge of a cutoff quantum number $K$ and energy $\varepsilon_{K}$ will be shown to be crucial to find the rigorous most probable distribution $f$ in Section 3 which job cannot be done in the cosmological context of Section 4 if $\hbar$ is dropped at the outset (in the classical phase space element $\left.\partial^{D} \vec{x} \partial^{D} \vec{p}\right)$.
TABLE 3

\begin{tabular}{lccccc}
\hline$\nu_{j}=$ & 0 & 1 & 2 & 3 & 4 \\
\hline$\Gamma\left(\nu_{j}+1\right)=v_{j} !=$ & 1 & 1 & 2 & 6 & 24 \\
$\ln \Gamma\left(v_{j}+1\right)=$ & 0 & 0 & 0.6932 & 1.7918 & 3.1781 \\
\hline
\end{tabular}

TABLE 4

\begin{tabular}{cccccc}
\hline$v_{j}$ & $\psi_{\text {num }}$ & $\psi_{\text {exact }}$ & $\nu$ & $\psi_{\text {num }}$ & $\psi_{\text {exact }}$ \\
\hline 0 & -0.563 & -0.577 & 0.2 & -0.278 & -0.289 \\
1 & 0.412 & 0.423 & 0.4 & -0.057 & -0.061 \\
2 & 0.929 & 0.923 & 0.6 & 0.115 & 0.126 \\
3 & 1.248 & 1.256 & 0.8 & 0.271 & 0.285 \\
4 & 1.533 & 1.506 & - & - & - \\
\hline
\end{tabular}

\section{B. Extension from Integer to Continuous $v$}

In this appendix, we carefully examine the numerical justification of some algebraic manipulations done on the combinatorial entropy $S$ of (10), (11).

\section{Factorials versus Gammas}

As is well known, $v_{j}$ ! identically equals $\Gamma\left(\nu_{j}+1\right)$ at all nonnegative integers $v_{j}=0,1,2,3,4, \ldots$ as seen from the second line of the following brief table. Its third line records the corresponding values of the natural logarithm $\ln \Gamma\left(\nu_{j}+1\right)$ to be used as the input in Table 3 .

\section{B.1. Numerical Differentiation}

Next, we address the subtle question of computing

$$
\psi_{\text {num }}(\nu) \equiv\left[\frac{\partial \ln \Gamma(\nu+1)}{\partial \nu}\right]_{\text {num }}: 0 \leq v \leq 4
$$

where the suffix "num" stands for "numerically" and the inequality $0 \leq v \leq 4$ implies that $v$ has become a continuous variable over a test range $[0,4]$. This is a problem of integer programming and we tackle it by adopting the following procedure.

(i) First, a finite-difference table was prepared using the above-mentioned data on $\ln \Gamma\left(\nu_{j}+1\right)$. (ii) Next, at several chosen integral/fractional values of $\nu$, (B.1) was computed employing an efficient package based on Markoff's version of Newton's interpolation differentiated [23, page 883]. (iii) Finally, comparison was made with the standard values of the digamma function [23, pages 258, 267, 272] obtained from the "exact" definition

$$
\psi_{\text {exact }}(\nu+1) \equiv \frac{\partial \ln \Gamma(\nu+1)}{\partial \nu}=\frac{1}{\Gamma(\nu+1)} \int_{0}^{\infty} d t t^{\nu} e^{-t} \ln t
$$

\section{B.2. Results}

The accompanying table shows that $\psi_{\text {num }}$ of (B.1) and $\psi$ exact of (B.2) agree within $1 \%$ to $5 \%$ at the input integer values $v=0,1,2,3,4$. We also see that their mutual agreement is 
good at the small fractional values of $v=0.2,0.4,0.6,0.8$. Therefore, taking derivatives of $\ln \Gamma(1+v)$ at all continuous values of $v$ (including $v \ll 1$ ) is mathematically justified in (13a) and (14a) (see Table 4).

\section{Acknowledgment}

The authors thank the Council of Scientific and Industrial Research (CSIR), New Delhi, India, for the financial support.

\section{References}

[1] K. Huang, Ed., Statistical Mechanics, John Wiley \& Sons, New York, NY, USA, 2nd edition, 1987.

[2] L. D. Landau, E. M. Lifshitz, and L. P. Pitaevski, Statistical Physics, Part 1, Pergamon, Oxford, UK, 1980.

[3] S. Chandrasekhar, Principles of Stellar Dynamics, Dover, New York, NY, USA, 1960.

[4] I. R. King, "The structure of star clusters. II. Steady-state velocity distributions," The Astronomical Journal, vol. 70, p. 376, 1965.

[5] J.-M. Lévy-Leblond, "Nonsaturation of gravitational forces," Journal of Mathematical Physics, vol. 10, no. 5, pp. 806-812, 1969.

[6] E. H. Lieb, "The stability of matter," Reviews of Modern Physics, vol. 48 , no. 4, pp. 553-569, 1976.

[7] D . Lynden-Bell and R. M. Lynden-Bell, "On the negative specific heat paradox," Monthly Notices of the Royal Astronomical Society, vol. 181, pp. 405-419, 1977.

[8] J. J. Binney and S. D. Tremaine, Galactic Dynamics, Princeton University Press, Princeton, NJ, USA, 1987.

[9] T. Padmanabhan, "Statistical mechanics of gravitating systems," Physics Report, vol. 188, no. 5, pp. 285-362, 1990.

[10] F. Ahmad, W. C. Saslaw, and N. I. Bhat, "Statistical mechanics of the cosmological many-body problem," The Astrophysical Journal, vol. 571, no. 2, pp. 576-584, 2002.

[11] B. Leong and W. C. Saslaw, "Gravitational binding, virialization, and the peculiar velocity distribution of the galaxies," The Astrophysical Journal, vol. 608, no. 2, pp. 636-646, 2004.

[12] V. J. Menon and D. C. Agrawal, "Method of most probable distribution: new solutions and results," PRAMANA: Journal of Physics, vol. 33, no. 4, pp. 455-465, 1989.

[13] V. J. Menon, R. K. Dubey, and D. N. Tripathi, "Stable galaxies of finite masses in the most probable distribution," Physica A, vol. 367 , pp. 269-275, 2006.

[14] I. R. King, "The structure of star clusters. I. An empirical density law," The Astronomical Journal, vol. 67, no. 8, pp. 471485, 1962.

[15] J. Th. van Loon, F. Van Leeuwen, B. Smalley, et al., "A spectral atlas of post-main-sequence stars in $\omega$ Centauri: kinematics, evolution, enrichment and interstellar medium," Monthly Notices of the Royal Astronomical Society, vol. 382, no. 3, pp. 1353-1374, 2007.

[16] R. V. D. R. Wooley and D. A. Robertson, "Studies in the equilibrium of globular clusters (II)," Monthly Notices of the Royal Astronomical Society, vol. 116, p. 288, 1956.

[17] L. Spitzer Jr. and R. Harm, "Evaporation of stars from isolated clusters," The Astrophysical Journal, vol. 127, pp. 544-550, 1958.

[18] R. W. Michie, "On the distribution of high energy stars in spherical stellar systems," Monthly Notices of the Royal Astronomical Society, vol. 125, p. 127, 1963.
[19] C. P. Wilson, "Dynamical models of elliptical galaxies," The Astronomical Journal, vol. 80, pp. 175-187, 1975.

[20] S. von Hoerner, "Internal structure of globular clusters," The Astrophysical Journal, vol. 125, p. 451, 1957.

[21] V. De Alfero and T. Regge, Potential Scattering, North-Holland, Amsterdam, The Netherlands, 1965.

[22] R. J. Newton, The Complex j-Plane, Benjamin, New York, NY, USA, 1964.

[23] M. Abramovitz and I. A. Stegun, Eds., Handbook of Mathematical Functions, Dover, New York, NY, USA, 1972.

[24] S. Ninkovic, "A globular-cluster model with variable mean mass of a single star," Bulletin de l'Observatoire Astronomique de Belgrade, no. 154, pp. 9-12, 1996.

[25] K. Holland, R. F. Jameson, S. Hodgkin, M. B. Davies, and D. Pinfield, "Praesepe- two merging clusters?" Monthly Notices of the Royal Astronomical Society, vol. 319, no. 3, pp. 956-962, 2000.

[26] L. Pauling and E. B. Wilson, Introduction to Quantum Mechanics, McGraw-Hill, New York, NY, USA, 1935.

[27] L. I. Schiff, Quantum Mechanics, McGraw-Hill, New York, NY, USA, 1968. 

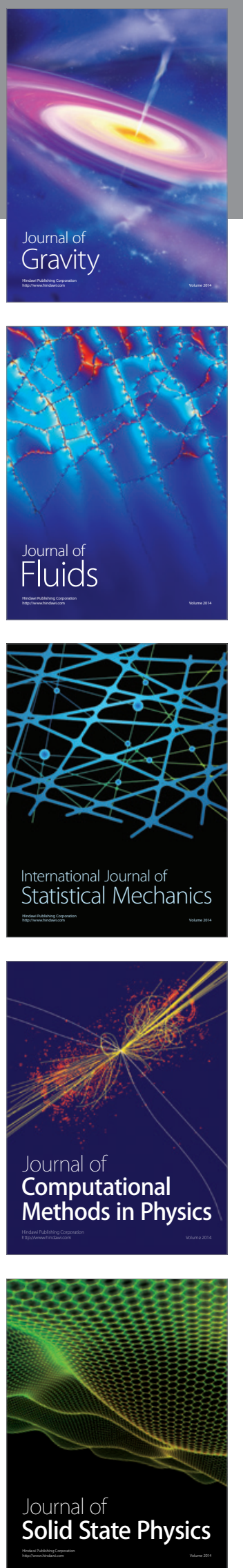

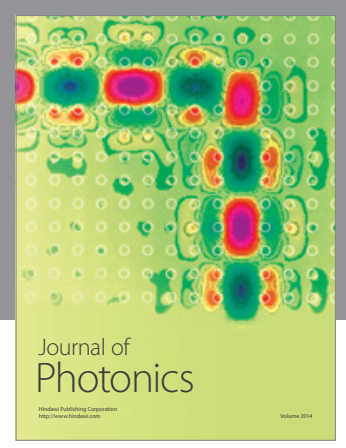

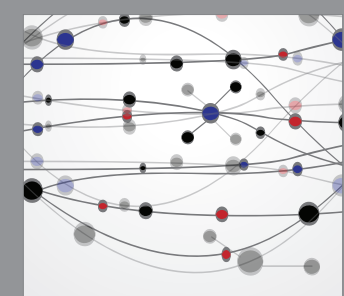

The Scientific World Journal
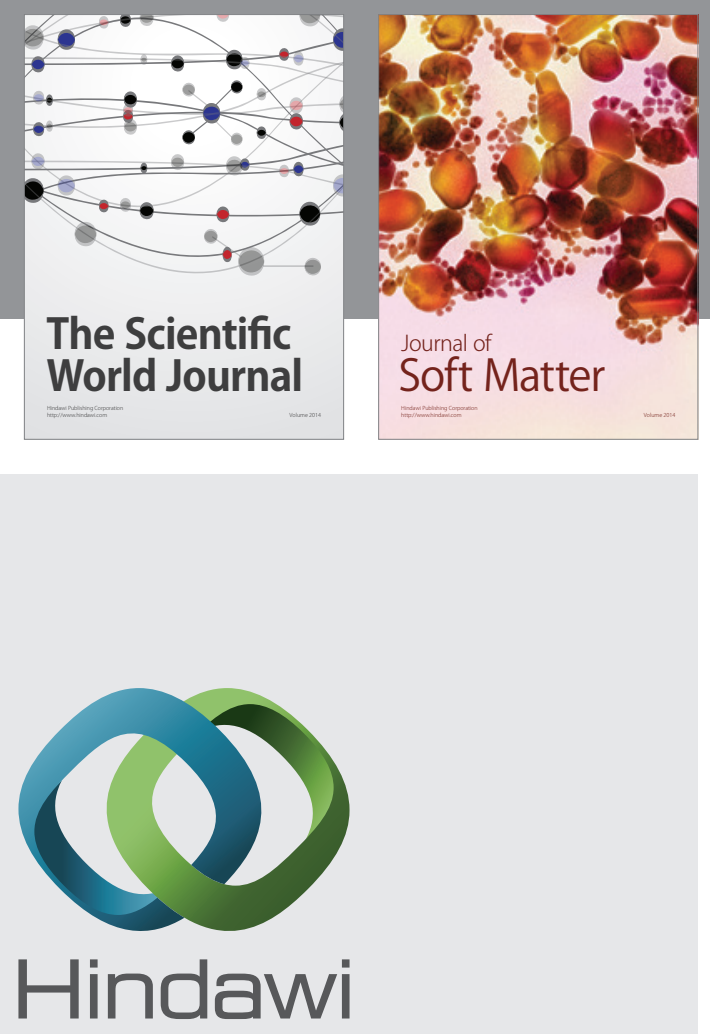

Submit your manuscripts at

http://www.hindawi.com
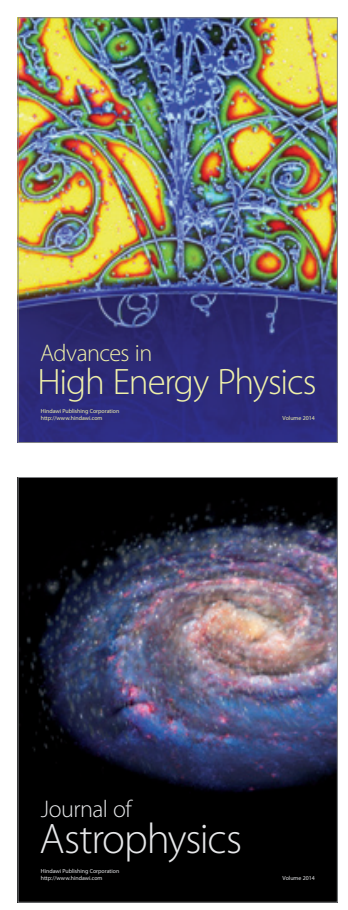
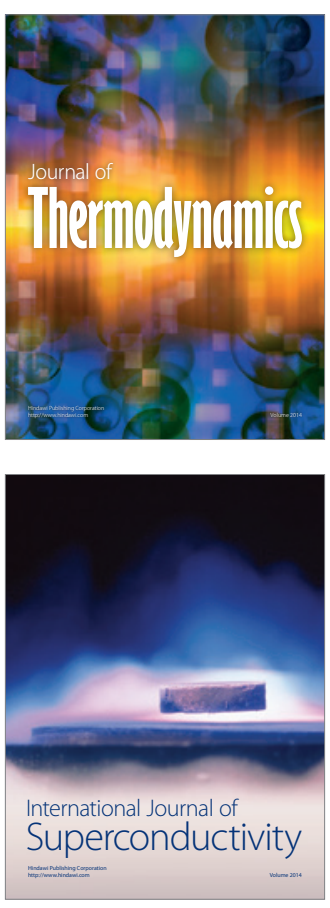
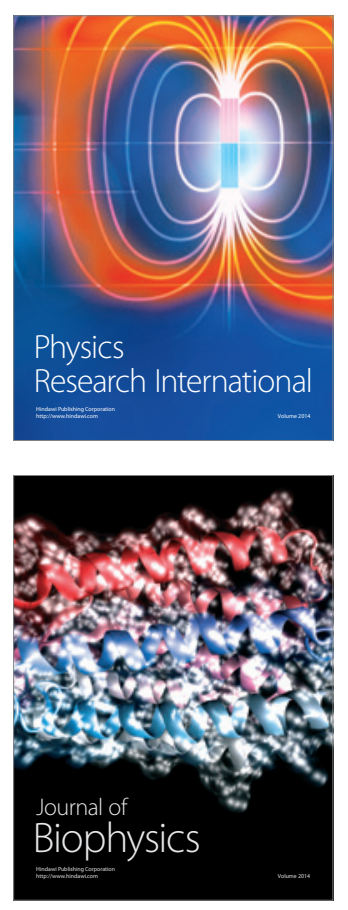
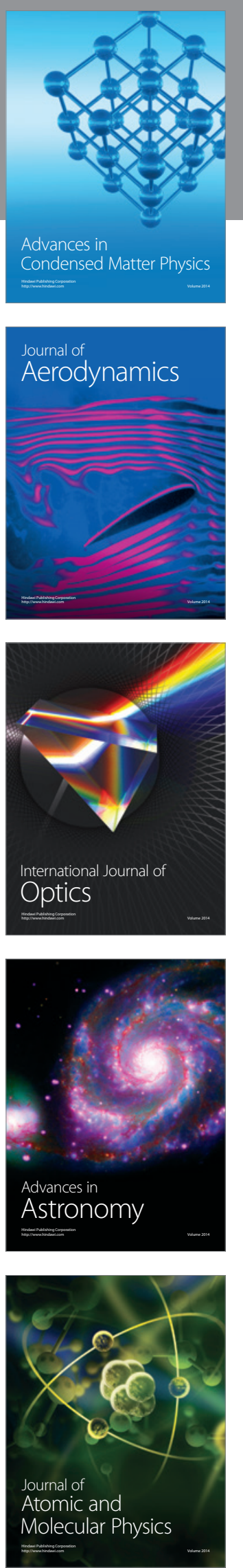